\title{
Genetics of response to cognitive behavior therapy in adults with major depression: a preliminary report
}

\author{
Evelyn Andersson ${ }^{1,2}$ - James J. Crowley ${ }^{1,3,4} \cdot$ Nils Lindefors $^{1,2}$ • Brjánn Ljótsson ${ }^{1,5}$ • Erik Hedman-Lagerlöf ${ }^{5}$. \\ Julia Boberg ${ }^{1,2} \cdot$ Samir El Alaoui ${ }^{1,2} \cdot{\text { Robert Karlsson } \mathbb{1}^{6} \cdot \text { Yi Lu }^{6} \cdot \text { Manuel Mattheisen }}^{1,2,7} \cdot$ Anna K. Kähler ${ }^{6}$. \\ Cecilia Svanborg ${ }^{1,2} \cdot$ David Mataix-Cols $^{1,2} \cdot$ Simon Mattsson $^{1,2} \cdot$ Erik Forsell $^{1,2} \cdot$ Viktor Kaldo $^{1,2,8} \cdot$ Martin Schalling $^{9}$. \\ Catharina Lavebratt $\mathbb{D}^{9} \cdot$ Patrick F. Sullivan ${ }^{3,4,6} \cdot$ Christian Rück $\mathbb{D}^{1,2}$
}

Published online: 8 November 2018

(c) The Author(s) 2018. This article is published with open access

\begin{abstract}
Major depressive disorder is heritable and a leading cause of disability. Cognitive behavior therapy is an effective treatment for major depression. By quantifying genetic risk scores based on common genetic variants, the aim of this report was to explore the utility of psychiatric and cognitive trait genetic risk scores, for predicting the response of 894 adults with major depressive disorder to cognitive behavior therapy. The participants were recruited in a psychiatric setting, and the primary outcome score was measured using the Montgomery Åsberg Depression Rating Scale-Self Rated. Single-nucleotide polymorphism genotyping arrays were used to calculate the genomic risk scores based on large genetic studies of six phenotypes: major depressive disorder, bipolar disorder, attention-deficit/hyperactivity disorder, autism spectrum disorder, intelligence, and educational attainment. Linear mixed-effect models were used to test the relationships between the six genetic risk scores and cognitive behavior therapy outcome. Our analyses yielded one significant interaction effect $(\mathrm{B}=0.09, p<0.001)$ : the autism spectrum disorder genetic risk score correlated with Montgomery Åsberg Depression Rating Scale-Self Rated changes during treatment, and the higher the autism spectrum disorder genetic load, the less the depressive symptoms decreased over time. The genetic risk scores for the other psychiatric and cognitive traits were not related to depressive symptom severity or change over time. Our preliminary results indicated, as expected, that the genomics of the response of patients with major depression to cognitive behavior therapy were complex and that future efforts should aim to maximize sample size and limit subject heterogeneity in order to gain a better understanding of the use of genetic risk factors to predict treatment outcome.
\end{abstract}

Electronic supplementary material The online version of this article (https://doi.org/10.1038/s41380-018-0289-9) contains supplementary material, which is available to authorized users.

$\triangle$ Christian Rück

Christian.ruck@ki.se

1 Centre for Psychiatry Research, Department of Clinical Neuroscience, Karolinska Institutet, Stockholm, Sweden

2 Stockholm Health Care Services, Stockholm County Council, Stockholm, Sweden

3 Center for Psychiatric Genomics, University of North Carolina, Chapel Hill, NC, USA

4 Department of Genetics, University of North Carolina, Chapel Hill, NC, USA

5 Division of Psychology, Department of Clinical Neuroscience,

\section{Introduction}

Major depressive disorder (MDD) is a common and heritable illness with a lifetime prevalence of around 14-15\%

Karolinska Institutet, Stockholm, Sweden

6 Department of Medical Epidemiology and Biostatistics, Karolinska Institutet, Stockholm, Sweden

7 Department of Biomedicine and Center for Integrated Sequencing (iSEQ), Aarhus University, Aarhus, Denmark

8 Department of Psychology, Faculty of Health and Life Sciences, Linnaeus University, Växjö, Sweden

9 Neurogenetics Unit, Department of Molecular Medicine and Surgery, Karolinska Institutet, Stockholm, Sweden; Center for Molecular Medicine, Karolinska University Hospital Solna, Stockholm, Sweden 
$[1,2]$. Twin studies have demonstrated that $\sim 40 \%$ of the variation in the liability to MDD is attributed to additive genetic effects [3]. However, the genetic foundations of MDD have long been unclear [4], although a recent genome-wide association study (GWAS) that included 130,664 cases reported 44 loci associated with MDD [5]. Cognitive behavior therapy (CBT), which is effective for MDD, is considered the treatment of choice for mild-tomoderate MDD according to international guidelines $[6,7]$. Unfortunately, access to CBT is limited to many patients due to cost, lack of trained therapists, and geographical barriers [8]. Internet-delivered CBT (iCBT) is an online form of therapist-guided CBT that is presented as a series of modules accompanied by homework assignments over multiple weeks [9]. iCBT has shown effect sizes comparable with traditional face- to-face CBT [10], and it may help bridge the supply and demand gap [11-15]. In addition, iCBT has the advantage of providing rich phenotypic information due to its highly monitored and manualized treatment protocol that often makes the progression of treatment contingent on filling out forms at different time points [16]. However, approximately half of the patients who undergo CBT do not respond [13]. Therefore, an important step toward identifying those who are more likely to respond to treatment is to distinguish the predictors of outcome to prevent patients from experiencing treatment failure.

Several studies have suggested clinical predictors of CBT outcome, such as baseline symptom severity [17], psychopathological comorbidity [18], having a social support network [19, 20], greater burden of illness [21-23], treatment adherence [24], and working full-time employment [25].

However, the results have been mixed [17]. Therefore, factors with acceptable predictive power to guide clinical decisions are not currently available [26].

The study of genetic predictors of psychological treatment outcome, sometimes called therapygenetics [27], is a fairly new field [28]. Similar to pharmacogenetics, the aim of therapygenetics is to use genetic data to better predict the outcome of psychological treatment and personalize interventions [29]. The earliest work in the field of therapygenetics has focused on candidate genes [27], but many of the findings were not replicable or inconsistent [30, 31], Because the effects of each genetic factor that influences therapy response are likely small and dispersed across the genome, these types of analyses should focus on genomewide variations rather than single polymorphisms. The first GWAS of CBT response, of 980 subjects with anxiety disorders did not detect any significant common variants [32]. However, an epigenetic study reported an association of monoamine oxidase A methylation with CBT response in individuals with panic disorder [33]. In addition, the results of a subsample in a recent genome-wide expression study suggested the association of a few genetic variants with exposure-based treatment response in 102 patients with panic disorder and specific phobias [34].

However, experience from pharmacogenetic studies implicates the unlikeliness of finding strongly replicated single-nucleotide polymorphisms (SNPs) with a large contribution to a complex trait, such as treatment response, and that large samples and well-defined homogeneous phenotypes are needed [35]. Attempts have therefore been made to aggregate the effects of common genetic variants to identify or explain a meaningful proportion of the genetic load of treatment response by calculating genetic risk score (GRS) [36], which quantifies the inherited burden of common variants across the genome for a given $p$-value threshold.

The present study explored the utility of GRSs for predicting treatment response in a sample of 894 subjects with MDD who underwent a standardized iCBT protocol. We tested the hypothesis that a GRS for MDD and five other psychiatric (bipolar disorder, ADHD and autism spectrum disorder) and cognitive traits (intelligence and educational attainment) are associated with the effects of iCBT treatment over time. We chose these traits because cause often informs cure [37], greater genetic risk for psychopathology could index severity that is suggested a predictor of poor outcome [38], and cognitive ability that has been associated with CBT treatment response [39]. To the best of our knowledge, this is the first study to fully investigate the genetic risks of psychological treatment response in MDD.

\section{Materials and methods}

\section{Subject characteristics}

Between 2008 and 2016, adult patients with MDD who started iCBT at the Internet Psychiatry Clinic in Stockholm [40], a government-funded psychiatric clinic specializing in delivering psychologist-guided iCBT, were asked to participate in the study. The treatment center is part of the public psychiatric care provided by the Stockholm County Council. The patients were asked to donate a blood sample for DNA. The patients had either been referred to the clinic by their general practitioner or via an online self-referral system. See Table 1 for a full description of the 894 study participants included in the final analysis. As detailed below, individuals from the original sample of 964 were excluded from the study for the following reasons: being an ancestry outlier $(n=49)$, quality control issues $(n=11)$, and missing phenotypes $(n=10)$.

After an online screening, the patients came to the clinic for psychiatric assessments, including a structured diagnostic interview (Mini-International Neuropsychiatric Interview) [41]. A psychiatrist or supervised psychiatry 
Table 1 Demographic characteristics of the participants

\begin{tabular}{|c|c|}
\hline$\overline{\text { Variable }}$ & Sample $^{1} N=894$ \\
\hline \multicolumn{2}{|l|}{ Gender } \\
\hline Women & $586(65.5 \%)$ \\
\hline Men & $308(34.5 \%)$ \\
\hline \multicolumn{2}{|l|}{ Age } \\
\hline Mean age (SD) & $37.9(11.8)$ \\
\hline Range & $18-75$ \\
\hline \multicolumn{2}{|l|}{ Occupational status } \\
\hline Working & $640(71.6 \%)$ \\
\hline \multicolumn{2}{|l|}{ Education } \\
\hline 7-9 years in school & $16(1.8 \%)$ \\
\hline Incomplete vocational or secondary school & $35(3.9 \%)$ \\
\hline Vocational school & $53(5.9 \%)$ \\
\hline Secondary school & $183(20.5 \%)$ \\
\hline University/college, uncompleted & $168(18.8 \%)$ \\
\hline University/college, completed & $437(48.9 \%)$ \\
\hline Other or unknown & $2(0.2 \%)$ \\
\hline \multicolumn{2}{|l|}{ Relationship status } \\
\hline Married or de facto & $493(55.1 \%)$ \\
\hline Single & $261(29.2 \%)$ \\
\hline Divorced & $135(15.1 \%)$ \\
\hline Widow/widower & $3(0.3 \%)$ \\
\hline Missing & $2(0.2 \%)$ \\
\hline \multicolumn{2}{|l|}{ Psychotropic medication } \\
\hline Previously (yes) & $372(41.6 \%)$ \\
\hline Currently (yes) & $514(57.5 \%)$ \\
\hline \multicolumn{2}{|l|}{ Comorbidity } \\
\hline Comorbid anxiety disorder & $202(22.6 \%)$ \\
\hline Comorbid other & $18(0.2 \%)$ \\
\hline \multicolumn{2}{|l|}{ MDD type at inclusion } \\
\hline Mild & $134(15.0 \%)$ \\
\hline Moderate & $193(21.6 \%)$ \\
\hline Severe & $2(0.2 \%)$ \\
\hline Recurrent mild & $170(19.0 \%)$ \\
\hline Recurrent moderate & $316(35.3 \%)$ \\
\hline Recurrent severe & $9(1.0 \%)$ \\
\hline Other & $70(8.0 \%)$ \\
\hline \multicolumn{2}{|l|}{ Suicide attempts } \\
\hline Previously (yes) & $52(5.8 \%)$ \\
\hline
\end{tabular}

$S D$ standard deviation, $M D D$ major depressive disorder

${ }^{1}$ All values are $n$ (\% of total) unless otherwise noted

resident performed the interview. For enrollment in the study, the patient had to meet the following requirements: fulfill the criteria in the DSM IV-TR for current MDD $[42,43]$, be able to read and write in Swedish, and be at least 18 -year-old. The exclusion criteria were any of the following: severe MDD combined with moderate to high risk of suicide, recent medication changes, comorbid bipolar or other psychotic disorder, unable to participate in concurrent psychotherapy, current alcohol or illicit drug abuse/dependence, or communication difficulties that impact treatment. The study was approved by the Regional Ethics Board in Stockholm, Sweden. All participants provided written informed consent.

\section{Intervention}

The core interventions of iCBT are the same as those administered face-to-face in conventional CBT. The iCBT program consisted of 10 text modules with components covering standard CBT interventions for patients with MDD, such as psychoeducation, cognitive restructuring, behavioral activation, and relapse prevention, that were to be completed in 12 weeks. Each module had a set of tasks and homework assignments to be completed each week that were monitored by the therapist via the secure online platform. In general, the patient and therapist interactions were limited to email contact, and there were no live meetings. A thorough description of the program has been published previously [44].

\section{Primary outcome measure}

The primary outcome measure was assessed using the Montgomery Åsberg Depression Rating Scale-Self report (MADRS-S) [45]. The MADRS-S total score, which ranges from 0 to 54, measures nine clinical characteristics of depression. The MADRS-S was assessed at treatment start (MADRS-S baseline), once each week during treatment, and in the last week of treatment (MADRS-S Post). Thus, each individual provided up to 12 weekly MADRS-S assessments that were included in the analyses. See Supplementary Table 2.

\section{Genotyping}

Genotyping was performed at LIFE \& BRAIN GmbH (Bonn, Germany) using the Infinium Global Screening Array 1.0 BeadArray (Illumina, Inc., San Diego, CA, USA) and automated workflow according to the manufacturer's instructions. The raw data were analyzed using GenomeStudio 2.0 (Illumina, Inc.) using the Infinium cluster file (GSA-24v1-0_A1_ClusterFile.egt). A reclustering step was performed using the GenTrain 3 algorithm in Genome Studio 2.0.

\section{Discovery datasets}

GRSs were generated for the following six phenotypes: MDD, bipolar disorder (BIP), attention-deficit/hyperactivity disorder (ADHD), autism spectrum disorder (ASD), 
intelligence (IQ), and educational attainment (EDU). We obtained the corresponding GWAS results for MDD, BIP [46], ADHD [47], and ASD [48] from the Psychiatric Genomics Consortium (PGC) website (https://www.med. unc.edu/pgc/results-and-downloads) and the GWAS results for IQ and EDU from published GWAS meta-analyses [49, 50]. The target set (currently studied iCBT samples) were not part of these previous GWAS meta-analyses.

\section{Target dataset}

The GWAS data from the 964 iCBT samples were processed using the PGC Ricopili pipeline for quality control and genotype imputation with reference genomes from the 1000 Genomes Project (phase 1 version 3) [51]. Eleven samples were excluded due to sample overlap (two pairs), cryptic relatedness (two pairs with pi-hat $\geq 0.2$ ), or poor call rate (three samples). After excluding 49 subjects due to nonEuropean ancestry, the top 20 ancestry principal components (PC) were calculated from the best-guess imputed genotypes, please see Supplementary Figure 1. Ten participants who failed to start treatment after inclusion were excluded due to missing phenotype data, resulting in a final sample total of 894 . The details of the SNP quality control of the discovery and target datasets and reference data, together with the overlapping numbers of SNPs among these three sets, are provided in Supplementary Figure 2.

\section{GRS calculation}

The GRS values were derived for the target set iCBT samples as the sum of the scores based on the risk alleles weighted by the effect size from the discovery sample. To select an independent set of SNPs for calculating the GRS, we conducted linkage disequilibrium clumping $\left(\mathrm{r}^{2}<0.1\right.$ in $1-\mathrm{Mb}$ window) on the overlapping SNPs using the European samples from the 1000 Genomes Project as a linkage disequilibrium reference. We computed eight sets of GRS for each phenotype under the $p$-value cutoffs of $\leq 1 \times 10^{-5}$, $\leq 1 \times 10^{-4}, \leq 0.001, \leq 0.01, \leq 0.05, \leq 0.1, \leq 0.5, \leq 1$. The GRS calculations were performed using PLINK (version 1.9) [52].

\section{Statistical analyses}

The statistical analyses were performed using $R$ [53]. To analyze the association between the six calculated GRS values and iCBT treatment outcome measured by MADRS$S$, we used the lme4 package [54] to perform full information maximum likelihood mixed models, including all available data for all patients. First, we fitted a model that determined the overall course of the MADRS-S values over the treatment period. This model included linear and quadratic effects of time (to allow for curvilinear development over time, which provided the best fit of the data) as fixed effects. The model also included a random intercept and random effect of time. Second, we investigated the influence of GRS on the rate of change during treatment. In all models, covariates (i.e., GRS) and possible confounders (i.e., ancestry PC scores, age, and sex) were added as both main effects and interaction effects with linear effect of time. The interpretation of a significant main effect of a GRS is that the GRS had a constant effect on the MADRS-S rating throughout the entire treatment period. The interpretation of a significant GRS $\times$ time interaction effect is that the GRS influenced the rate of improvement during treatment. These analyses were performed in the following steps: (1) Each of the six GRS domains at the predetermined $p$-value cutoff were investigated in separate models while controlling for the top five ancestry PC scores. (2) Age and sex were added to the models in step 1. (3) A full model was created in which all six GRSs were entered while controlling for ancestry PCs, age, and sex. As stated above, all covariates (GRS scores, ancestry PCs, age, and sex) were entered as both main effects and interaction effects with linear time in these analyses. To reduce multiple testing, we tested each of the six GRS at predetermined $p<0.05$ in main analyses. In addition, we presented the results on GRS at all $p$-value thresholds as sensitivity analyses (Supplementary Table 1).

\section{Outlier analyses}

We performed outlier analyses to detect influential cases that may have biased the regression models. These analyses were performed on the GRS $p<0.05$ models (controlling for PC scores, age, and sex) with which significant or nearsignificant $(p<0.10)$ main or interaction effects were obtained. For this, we used the influence.ME package [55] to calculate Cook's distance for all observations (i.e., one MADRS-S rating) and all individuals (i.e., all MADRS-S ratings by one individual). Possible influential observations and individuals were identified by visual inspection of the Cook's distance plots, and the regression analyses were rerun with the outlying observations or individuals removed. Removing influential observations or individuals did not result in altered interpretations of the significant or nearsignificant results in any of the cases.

\section{Results}

\section{Treatment effects}

We observed a significant negative effect of time $\left(\mathrm{B}=-1.29, p<2 \times 10^{-16}\right)$ and a significant positive effect 
of quadratic time $\left(\mathrm{B}=0.048, p<2 \times 10^{-16}\right)$ on MADRS-S ratings. See Supplementary Table 2 for mean weekly ratings on the MADRS-S for the whole group. These results indicated that the patients' depression scores decreased during treatment, with larger declines in the beginning.

\section{Effects of GRS on treatment response}

The first step in our analyses (GRS with $p<0.05$ scores while controlling for ancestry PC scores) yielded one significant result. Namely, the ASD GRS was associated with MADRS-S changes over treatment time. This was reflected in the significant interaction effect $(\mathrm{B}=0.09, p<.001)$ between ASD GRS and time (GRS $\times$ time): meaning that the higher the ASD genetic load, the less iCBT treatment response over time (Supplementary Table 1 and Fig. 1). This result remained significant after correcting for multiple testing. None of the other individual traits that were examined had a GRS with consistent significant effects on the MADRS-S scores or changes in MADRS-S scores over time.

\section{Discussion}

This is the first study to explore the utility of GRS for predicting response to psychological treatment for patients with MDD. We set out to test the hypothesis that the GRS for six psychiatric and cognitive traits would be associated with treatment outcome. Participants with the highest ASD GRS showed a poorer response to treatment versus those with average or low ASD GRS.

The finding that ASD GRS was associated with outcome suggested that autism spectrum related genetic risk also puts one at risk for failing to respond to cognitive behavioral treatment for depression. If we assume that a high ASD GRS is associated with greater expression of autism spectrum phenotypes [56], we can speculate why ASD traits are related to worse MDD treatment outcome. For example, patients with high ASD GRS may have depressive symptoms due to ASD-related difficulties, such as problems with social communication and interaction, rather than phenotypes associated with MDD, such as distortive negative current thoughts and lack of reinforcing behavior. Consequently, iCBT for MDD would not target the main reasons for the depressive state in patients with higher load for ASD GRS, which could lead to even more negative emotions and feelings of failure. Furthermore, perhaps patients with a high ASD GRS load may have greater difficulty identifying with the rationale of the psychoeducational part of the therapy and thereby increase the risk of poorer response to the treatment.

Notably, MDD GRS was not associated with treatment outcome, and there are multiple possible explanations. First, the cause of depression and the treatment of depression may

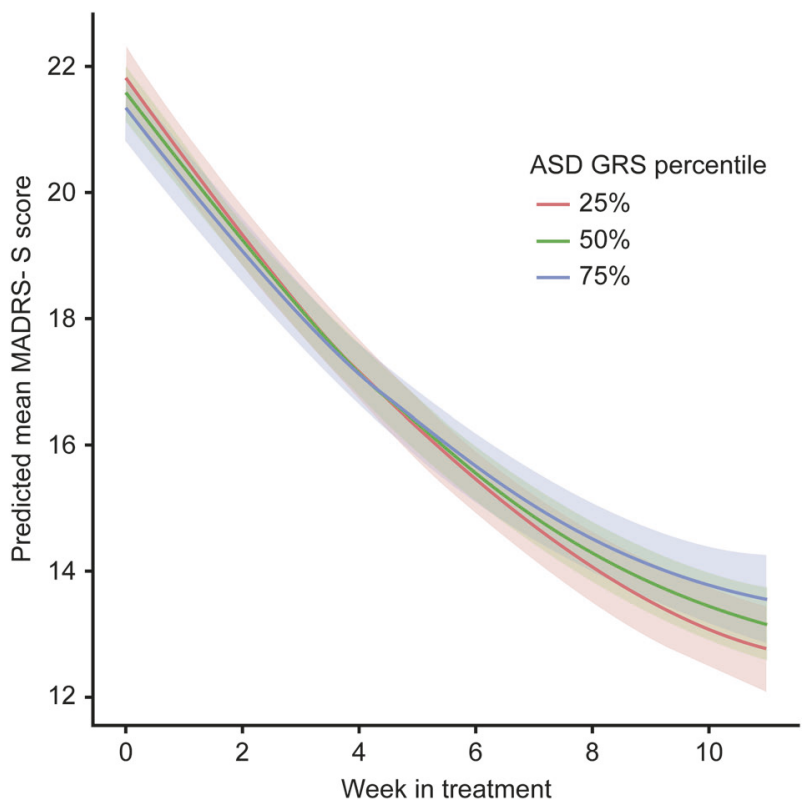

Fig. 1 Effects of ASD GRS (at a $p$-value threshold of 0.05) on MADRS-S scores during iCBT treatment. The figure shows the predicted MADRS-S score for every week during treatment for three different levels of the ASD GRS (25th, 50th, and 75th percentiles). The shaded areas show the $95 \%$ confidence intervals of the predicted values. The participants with the highest ASD GRS scores (blue) showed poorer responses to treatment vs. those with average (green) or low (red) ASD GRS scores. Abbreviations: autism spectrum disorder (ASD), genetic risk score (GRS), Montgomery Åsberg Depression rating scale-Self (MADRS-S), internet-delivered cognitive behavior therapy (iCBT)

involve different genetic factors. In other words, MDD GRS might be related to why a person gets MDD but not related to the treatment response of MDD. Second, because we excluded patients with severe MDD, we may have selected for overall lower MDD GRS values. Finally, the GRS training set may not have been large enough.

The strengths of this study include the largest yet sample size in a genetic study focused on psychological therapy treatment response for MDD. Because all patients were diagnosed using a structured interview by a psychiatrist or supervised resident doctor suggests that the MDD phenotype was reliable. The iCBT treatment allows for tight control of what treatment was delivered with minimal risk for therapist or patient drift away from the treatment protocol. In addition, the study participants completed wellvalidated outcome measures at 12 time points (including pre- and post-assessments), thus producing a large body of treatment outcome data. The limitations of the study include that this study was likely still too small to detect robust and reliable associations with treatment response on both the aggregate (GRS) and individual locus (SNP) levels. In addition, the therapeutic mechanisms of iCBT can differ from traditional CBT and hereby limit the generalization of the results. 
Our finding of an association of ASD GRS with CBT outcome is the first significant finding using a genome-wide approach in the field of therapygenetics. These preliminary findings need to be replicated before firm conclusions can be drawn and the possibility of the finding being a false positive must be considered.

Acknowledgements This study was supported and enabled by the efforts of psychiatric research nurse Monica Hellberg, operations manager Nina Lind, and the staff at the Internet psychiatry clinic at Stockholm County Council. Christian Rück and Patrick F. Sullivan gratefully acknowledge support from the Swedish Research Council (Vetenskapsrådet, award CO250103 and D0886501, respectively).

\section{Compliance with ethical standards}

Conflict of interest PFS reports the following potentially competing financial interests: Current, Lundbeck (advisory committee, grant recipient); Past three years, Pfizer (scientific advisory board), Element Genomics (consultation fee), and Roche (speaker reimbursement). Remaining authors declare no financial interests or potential conflicts of interest.

Open Access This article is licensed under a Creative Commons Attribution 4.0 International License, which permits use, sharing, adaptation, distribution and reproduction in any medium or format, as long as you give appropriate credit to the original author(s) and the source, provide a link to the Creative Commons license, and indicate if changes were made. The images or other third party material in this article are included in the article's Creative Commons license, unless indicated otherwise in a credit line to the material. If material is not included in the article's Creative Commons license and your intended use is not permitted by statutory regulation or exceeds the permitted use, you will need to obtain permission directly from the copyright holder. To view a copy of this license, visit http://creativecommons. org/licenses/by/4.0/.

\section{References}

1. Kessler RC, Berglund P, Demler O, Jin R, Koretz D, Merikangas $\mathrm{KR}$, et al. The epidemiology of major depressive disorder: results from the National Comorbidity Survey Replication (NCS-R). JAMA. 2003;289:3095-105.

2. Kessler RC, Bromet EJ. The epidemiology of depression across cultures. Annu Rev Public Health. 2013;34:119-38.

3. Sullivan PF, Neale MC, Kendler KS. Genetic epidemiology of major depression: review and meta-analysis. Am J Psychiatry. 2000;157:1552-62.

4. Levinson DF, Mostafavi S, Milaneschi Y, Rivera M, Ripke S, Wray NR, et al. Genetic studies of major depressive disorder: why are there no genome-wide association study findings and what can we do about it? Biol Psychiatry. 2014;76:510-2.

5. Wray NR, Ripke S, Mattheisen M, Trzaskowski M, Byrne EM, Abdellaoui A, et al. Genome-wide association analyses identify 44 risk variants and refine the genetic architecture of major depression. Nat Genet. 2018;50:668-81.

6. Cuijpers P, Berking M, Andersson G, Quigley L, Kleiboer A, Dobson KS. A meta-analysis of cognitive-behavioural therapy for adult depression, alone and in comparison with other treatments. Can J Psychiatry. 2013;58:376-85.

7. National Institute for Health and Clinical Excellence: Guidance. Depression: The Treatment and Management of Depression in Adults (Updated Edition): Leicester (UK), 2010.
8. Cavanagh K. Geographic inequity in the availability of cognitive behavioural therapy in England and Wales: a 10-year update. Behav Cogn Psychother. 2014;42:497-501.

9. Hedman E. Therapist guided internet delivered cognitive behavioural therapy. BMJ. 2014;348:g1977.

10. Carlbring P, Andersson G, Cuijpers P, Riper H, Hedman-Lagerlof E. Internet-based vs. face-to-face cognitive behavior therapy for psychiatric and somatic disorders: an updated systematic review and meta-analysis. Cogn Behav Ther. 2018;47:1-18.

11. Andersson G, Bergstrom J, Hollandare F, Carlbring P, Kaldo V, Ekselius L. Internet- based self-help for depression: randomised controlled trial. Br J Psychiatry. 2005;187:456-61.

12. Andersson G, Carlbring P. Internet and cognitive behaviour therapy: new opportunities for treatment and assessment. Cogn Behav Ther. 2003;32:97-99

13. Andersson G, Cuijpers P, Carlbring P, Riper H, Hedman E. Guided internet-based vs. face-to-face cognitive behavior therapy for psychiatric and somatic disorders: a systematic review and metaanalysis. World Psychiatry. 2014;13:288-95.

14. Andersson G, Rozental A, Shafran R, Carlbring P. Long-term effects of internet-supported cognitive behaviour therapy. Expert Rev Neurother. 2018;18:21-8.

15. Bergstrom J, Andersson G, Ljotsson B, Ruck C, Andreewitch S, Karlsson A, et al. Internet-versus group-administered cognitive behaviour therapy for panic disorder in a psychiatric setting: a randomised trial. BMC Psychiatry. 2010;10:54.

16. Andersson G. Using the Internet to provide cognitive behaviour therapy. Behav Res Ther. 2009;47:175-80.

17. Eskildsen A, Hougaard E, Rosenberg NK. Pre-treatment patient variables as predictors of drop-out and treatment outcome in cognitive behavioural therapy for social phobia: A systematic review. Nord J Psychiatry. 2010;64:94-105.

18. Stiles-Shields C, Kwasny MJ, Cai X, Mohr DC. Comorbid anxiety as a differential treatment predictor for telephone versus face-toface administered cognitive behavioral therapy for depression. Depress Anxiety. 2014;31:934-40.

19. Jarrett RB, Eaves GG, Grannemann BD, Rush AJ. Clinical, cognitive, and demographic predictors of response to cognitive therapy for depression: a preliminary report. Psychiatry Res. 1991;37:245-60.

20. Sotsky SM, Glass DR, Shea MT, Pilkonis PA, Collins JF, Elkin I, et al. Patient predictors of response to psychotherapy and pharmacotherapy: findings in the NIMH treatment of depression collaborative research program. Am J Psychiatry. 1991;148:9971008.

21. Simons AD, Gordon JS, Monroe SM, Thase ME. Toward an integration of psychologic, social, and biologic factors in depression: effects on outcome and course of cognitive therapy. J Consult Clin Psychol. 1995;63:369-77.

22. Thase ME, Simons AD, Cahalane J, McGeary J, Harden T. Severity of depression and response to cognitive behavior therapy. Am J Psychiatry. 1991;148:784-9.

23. Hoberman HM, Lewinsohn PM, Tilson M. Group treatment of depression: individual predictors of outcome. J Consult Clin Psychol. 1988;56:393-8.

24. El Alaoui S, Ljotsson B, Hedman E, Svanborg C, Kaldo V, Lindefors N. Predicting outcome in internet-based cognitive behaviour therapy for major depression: a large cohort study of adult patients in routine psychiatric care. PLoS ONE. 2016;11: e0161191.

25. Hedman E, Andersson E, Ljotsson B, Andersson G, Andersson E, Schalling $\mathrm{M}$, et al. Clinical and genetic outcome determinants of Internet- and group-based cognitive behavior therapy for social anxiety disorder. Acta Psychiatr Scand. 2012;126: 126-36. 
26. Schneider RL, Arch JJ, Wolitzky-Taylor KB. The state of personalized treatment for anxiety disorders: a systematic review of treatment moderators. Clin Psychol Rev. 2015;38:39-54.

27. Eley TC, Hudson JL, Creswell C, Tropeano M, Lester KJ, Cooper P, et al. Therapygenetics: the 5HTTLPR and response to psychological therapy. Mol Psychiatry. 2012;17:236-7.

28. Lester KJ, Eley TC. Therapygenetics: using genetic markers to predict response to psychological treatment for mood and anxiety disorders. Biol Mood Anxiety Disord. 2013;3:4.

29. Eley TC. The future of therapygenetics: where will studies predicting psychological treatment response from genomic markers lead? Depress Anxiety. 2014;31:617-20.

30. Lester KJ, Roberts S, Keers R, Coleman JR, Breen G, Wong CC, et al. Non-replication of the association between 5HTTLPR and response to psychological therapy for child anxiety disorders. Br J Psychiatry. 2016;208:182-8.

31. Andersson E, Rück C, Lavebratt C, Hedman E, Schalling M, Lindefors N, et al. Genetic polymorphisms in monoamine systems and outcome of cognitive behavior therapy for social anxiety disorder. PLoS ONE. 2013;8:e79015.

32. Coleman JR, Lester KJ, Keers R, Roberts S, Curtis C, Arendt K, et al. Genome-wide association study of response to cognitivebehavioural therapy in children with anxiety disorders. Br J Psychiatry. 2016;209:236-43.

33. Ziegler C, Richter J, Mahr M, Gajewska A, Schiele MA, Gehrmann A, et al. MAOA gene hypomethylation in panic disorderreversibility of an epigenetic risk pattern by psychotherapy. Transl Psychiatry. 2016;6:e773.

34. Roberts S, Wong CCY, Breen G, Coleman JRI, De Jong S, Johren $\mathrm{P}$, et al. Genome-wide expression and response to exposure-based psychological therapy for anxiety disorders. Transl Psychiatry. 2017;7:e1219.

35. Garcia-Gonzalez J, Tansey KE, Hauser J, Henigsberg N, Maier W, Mors $\mathrm{O}$, et al. Pharmacogenetics of antidepressant response: a polygenic approach. Prog Neuropsychopharmacol Biol Psychiatry. 2017;75:128-34.

36. Demirkan A, Penninx BW, Hek K, Wray NR, Amin N, Aulchenko YS, et al. Genetic risk profiles for depression and anxiety in adult and elderly cohorts. Mol Psychiatry. 2011;16:773-83.

37. Uher R. The implications of gene-environment interactions in depression: will cause inform cure? Mol Psychiatry. 2008;13:1070-8.

38. Hoyer J, Wiltink J, Hiller W, Miller R, Salzer S, Sarnowsky S, et al. Baseline patient characteristics predicting outcome and attrition in cognitive therapy for social phobia: results from a large multicentre trial. Clin Psychol Psychother. 2016;23:35-46.

39. Renaud J, Russell JJ, Myhr G. Predicting who benefits most from cognitive-behavioral therapy for anxiety and depression. J Clin Psychol. 2014;70:924-32.

40. Hedman E, Ljotsson B, Ruck C, Bergstrom J, Andersson G, Kaldo $\mathrm{V}$, et al. Effectiveness of internet-based cognitive behaviour therapy for panic disorder in routine psychiatric care. Acta Psychiatr Scand. 2013;128:457-67.

41. Sheehan DV, Lecrubier Y, Sheehan KH, Amorim P, Janavs J, Weiller E, et al. The mini- international neuropsychiatric interview
(M.I.N.I.): the development and validation of a structured diagnostic psychiatric interview for DSM-IV and ICD-10. J Clin Psychiatry. 1998;59(Suppl 20):22-33. quiz 34-57

42. American Psychiatric Association. DSM-IV-TR. Diagnostic and Statistical Manual of Mental Disorders, 4th Edition. DSM-IV-TR 2000.

43. American Psychiatric Association. DSM-5 Diagnostic Classification. Diagnostic and Statistical Manual of Mental Disorders. 2013.

44. Hedman E, Ljotsson B, Kaldo V, Hesser H, El Alaoui S, Kraepelien M, et al. Effectiveness of internet-based cognitive behaviour therapy for depression in routine psychiatric care. J Affect Disord. 2014;155:49-58.

45. Svanborg P, Asberg M. A new self-rating scale for depression and anxiety states based on the comprehensive psychopathological rating scale. Acta Psychiatr Scand. 1994;89:21-8.

46. Stahl E, Forstner A, McQuillin A, Ripke S, Ophoff R, Scott L et al. Genomewide association study identifies 30 loci associated with bipolar disorder. bioRxiv. 2017;173062; https://doi.org/10. 1101/173062.

47. Demontis D, Walters RK, Martin J, Matheisen M, Damm Als T, Agerbo E et al. Discovery Of The First Genome-Wide Significant Risk Loci For ADHD. bioRxiv 145581; https://doi.org/10.1101/ 145581.

48. Autism Spectrum Disorders Working Group of The Psychiatric Genomics C. Meta- analysis of GWAS of over 16,000 individuals with autism spectrum disorder highlights a novel locus at 10q24.32 and a significant overlap with schizophrenia. Mol Autism. 2017;8:21.

49. Okbay A, Beauchamp JP, Fontana MA, Lee JJ, Pers TH, Rietveld CA, et al. Genome-wide association study identifies 74 loci associated with educational attainment. Nature. 2016;533:539-42.

50. Sniekers S, Stringer S, Watanabe K, Jansen PR, Coleman JRI, Krapohl E, et al. Genome-wide association meta-analysis of 78,308 individuals identifies new loci and genes influencing human intelligence. Nat Genet. 2017;49:1107-12.

51. 1000 Genomes Project Consortium, Auton A, Brooks LD, Durbin RM, Garrison EP, Kang HM, et al. A global reference for human genetic variation. Nature. 2015;526:68-74.

52. Purcell S, Neale B, Todd-Brown K, Thomas L, Ferreira MA, Bender D, et al. PLINK: a tool set for whole-genome association and population-based linkage analyses. Am J Hum Genet. 2007;81:559-75.

53. R: a language and environment for statistical computing. $R$ Foundation for Statistical Computing. http://www.R-project.org, 2016, Accessed on December 24, 2016.

54. Bates D, Mächler M, Bolker B, Walker S. Fitting Linear MixedEffects Models Using lme4. Journal of Statistical Software 2015;67:1-48.

55. MtG RenseNieuwenhuis. Ben Pelzer influence.ME: tools for detecting influential data in mixed effects models. R J. 2012; 4:38-47.

56. Guo W, Samuels JF, Wang Y, Cao H, Ritter M, Nestadt PS, et al. Polygenic risk score and heritability estimates reveals a genetic relationship between ASD and OCD. Eur Neuropsychopharmacol. 2017;27:657-66. 